\title{
Symmetric Negative Differential Resistance in a Molecular Nanosilver Chain
}

\author{
Tae Kyung Kim, ${ }^{1}$ Hoi Ri Moon, ${ }^{1}$ and Byung Hoon $\mathrm{Kim}^{2}$ \\ ${ }^{1}$ Interdisciplinary School of Green Energy, Ulsan National Institute of Science and Technology (UNIST), \\ Ulsan 689-798, Republic of Korea \\ ${ }^{2}$ Department of Physics, Incheon National University, Incheon 406-772, Republic of Korea
}

Correspondence should be addressed to Byung Hoon Kim; kbh37@incheon.ac.kr

Received 22 October 2013; Accepted 30 October 2013

Academic Editor: Mun Seok Jeong

Copyright (C) 2013 Tae Kyung Kim et al. This is an open access article distributed under the Creative Commons Attribution License, which permits unrestricted use, distribution, and reproduction in any medium, provided the original work is properly cited.

The electrical transport properties of the molecular nanosilver chain have been investigated. We observed the symmetric negative differential resistance (NDR) in the current-voltage characteristics. The peak voltage $\left(V_{P}\right)$ increased but the peak current $\left(I_{P}\right)$ decreased upon cooling. The self-capacitance effect of the silver chain crystal is suggested to explain this unconventional NDR phenomenon.

\section{Introduction}

Negative differential resistance (NDR) has attracted much interest due to its applications for switch, memory, and high-speed logic devices. NDR explained by a resonance tunneling diode has been shown in various materials, such as germanium $\mathrm{p}-\mathrm{n}$ junction [1], various heterostructures [26], semiconductor superlattices [7], self-assembled layered nanostructures [8], carbon atomic wires [9], mesoscopic manganite structures [10], and molecular electronics device systems [11-13]. The NDR has also been observed in graphene nanoribbon with unsaturated edges due to the strong current polarization [14] and due to the interaction between the narrow density of state of doped sites and the discrete states in the scattering region [15]. In addition, Ag-based materials, such as $\mathrm{Ag} / \mathrm{Si}$ nanowires on silicon carbide, exhibited NDR as well [16]. NDR phenomena in general, including those mentioned above, exhibited asymmetric current-voltage ( $I$ $V)$ characteristics with respect to the applied voltage.

Recently, Moon et al. reported a stair-shaped $\mathrm{Ag}^{0}$ coordination compound without bridging ligands (nanosilver chain) [17]. This nanosilver chain (NSC) consists of Ag atoms linked by covalent bonds and pyridine molecules. Herein, we report the abnormal $\mathrm{N}$-shaped NDR phenomenon of the single crystalline NSC and temperature-dependent NDR behavior that cannot be interpreted by previously reported mechanisms. The $I-V$ characteristics in the backward voltage sweep were symmetric to that in the forward voltage sweep. Hysteretic behavior was manifested as well. The peak voltage $\left(V_{P}\right)$ increased and the peak current $\left(I_{P}\right)$ decreased as the temperature decreased. We suggest that the NDR phenomenon of the NSC may be caused by a capacitance effect of the NSC itself.

\section{Materials and Methods}

The I-V characteristics were measured with the conventional 2-probe method. Silver paint (DuPont 4929N) and carbon paint (Dotite XC-12) contacts were made to 0.01 inch diameter gold wires. The $I-V$ characteristics were doubly checked using semiconductor characterization systems (Keithley 4200-SCS) and electrometer (Keithley 6517A). The voltage sweep rate was $10 \mathrm{mV} / \mathrm{sec}$. The temperaturedependent $I-V$ characteristics were investigated with a cryogenic displex-osp.

\section{Results and Discussion}

The (001) and (010) planes of the single crystalline NSC are shown in Figure 1(a). The $I-V$ characteristics of the NSC crystal at room temperature measured in air right after mounting 


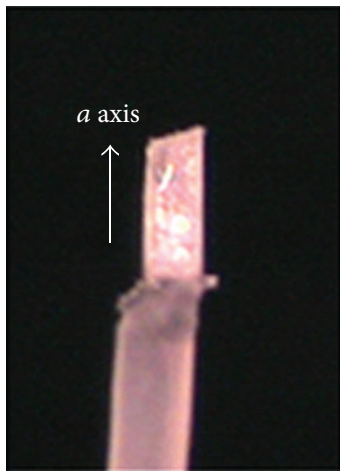

(001) plane

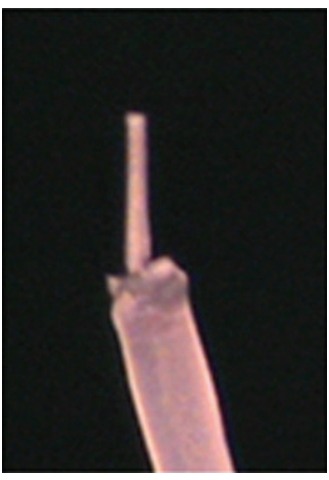

(010) plane

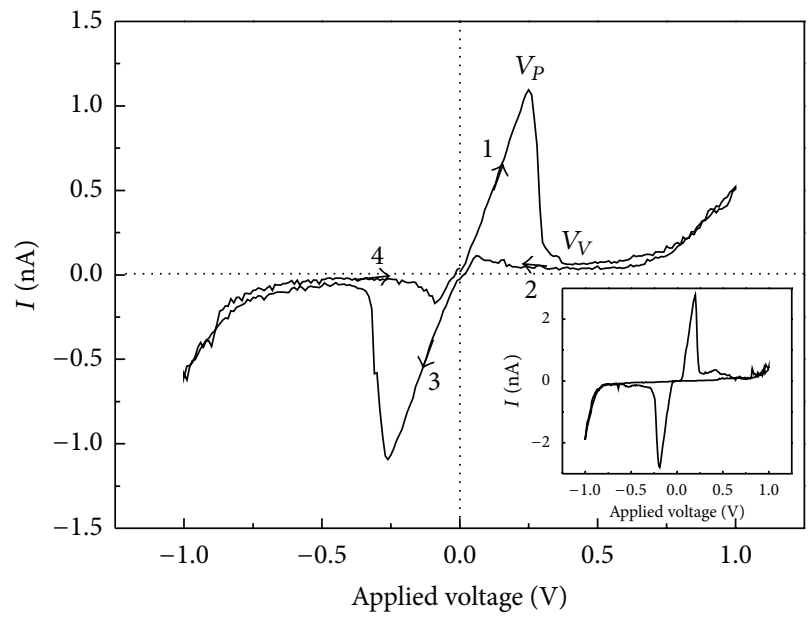

(b)

FIGURE 1: NDR behavior at room temperature. (a) An NSC single crystal is shown in the white box. (b) $I$ - $V$ curve is measured in air at $300 \mathrm{~K}$ by the conventional 2-probe method with DuPont, silver composition $4929 \mathrm{~N}$ paint. $I_{P}$ is a peak current. $V_{P}$ and $V_{V}$ are voltages corresponding to the peak and the valley of the current, respectively. The number shows the voltage sweep procedure. The $I-V$ curve with a carbon paint contact is shown in the inset.

the sample were the same as those measured in a vacuum after loading the sample in the displex cryogenic system. This implies that the NSC crystal lost pyridine molecules as soon as it was exposed to air [17] for the measurements. $\mathrm{N}$ type NDR was observed for $V>V_{P}(0.25 \mathrm{~V})$ as shown in Figure 1(b). Here, $V_{P}$ and $V_{V}$ are voltages corresponding to the peak and the valley of the current, respectively. The $I-V$ curve was linear in the low voltage region $\left(0<V<V_{P}\right)$. The current abruptly dropped at $V_{P}$ revealing NDR and started to increase gradually for $V>V_{V}$ (process "1"). In the backward sweep from high to low voltage, the current followed the same curve as that in the forward sweep for $V>V_{V}$. However, it exhibited a big hysteresis for $0<V<V_{V}$ with a significantly reduced NDR peak (process " 2 "). The $I-V$ characteristics of the negative voltage region (processes " 3 " and " 4 ") were symmetrical with those of the positive voltage region. To check the contact effect, the identical experiment with a carbon paint contact was also done. The $I-V$ characteristics with carbon paint were the same with a silver paint contact although the details were not identical (the inset of Figure 1). This symmetric NDR with respect to the applied voltage has never been reported. It will be discussed later.

The temperature-dependent $I-V$ characteristics of the NSC crystal from $230 \mathrm{~K}$ to $300 \mathrm{~K}$ are shown in Figure 2. All $I-V$ curves showed the symmetric NDR behavior and the data were reproducible for 3-5 cycles of $I-V$ measurements at each temperature. The peak current $\left(I_{P}\right)$ decreased but $V_{P}$ increased upon cooling. This is another unusual behavior in NDR phenomena. The resistivity $(\rho)$ at each temperature was obtained from the slope of the $I-V$ curve in the linear region $\left(V<V_{P}\right)$ (Figure $3(\mathrm{a})$ ). The resistivity at $300 \mathrm{~K}$ is $\rho_{300 \mathrm{~K}} \simeq 1.5 \times 10^{5} \Omega \mathrm{cm}$, which is in the range of resistivity for ionic solids. The various Ag-based materials follow ionic conduction [18-21]. Although no mobile ions are reportedly expected to exist in the NSC crystal [17], it is possible for

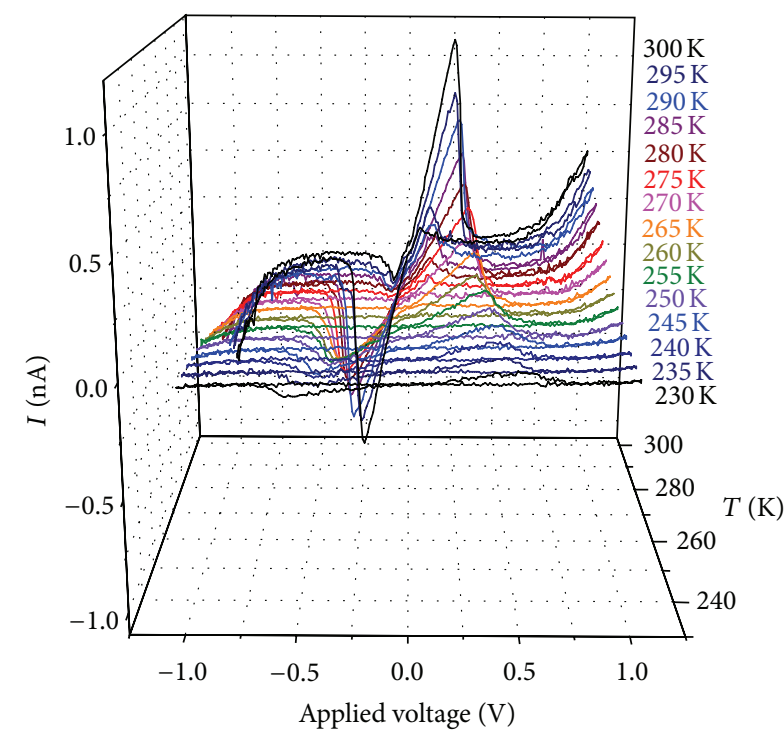

FIgure 2: Temperature-dependent NDR behavior. All $I$ - $V$ curves measured at various temperatures exhibit the symmetric NDR behavior. $V_{P}$ increases but $I_{P}$ decreases as the temperature decreases.

ionic defects to exist in the NSC crystal, and the defects can cause the current to flow through the sample by ionic conduction. The temperature-dependent conductivity of the NSC crystal seems to follow the ionic conduction behavior with the activation energy $0.35 \mathrm{eV}$. However, the resistivity is well fitted to the thermally activated hopping conduction, $\rho=\rho_{0} \exp \left(E_{a} / K_{B} T\right)$, rather than the ionic conduction as shown in the inset of Figure 3(a). $E_{a}$ and $K_{B}$ are the activation energy and the Boltzmann constant, respectively. Moreover, the ionic conduction mechanism does not explain the unusual temperature dependence of the symmetric NDR 


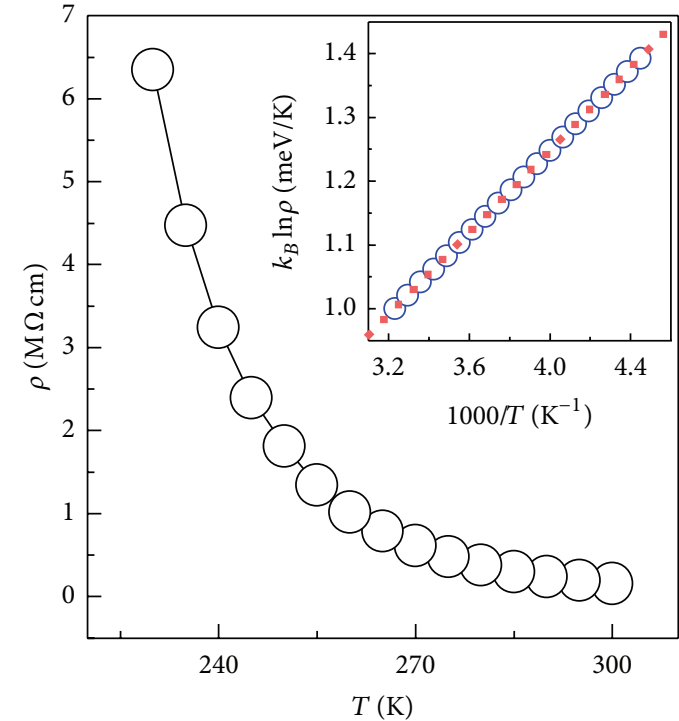

(a)

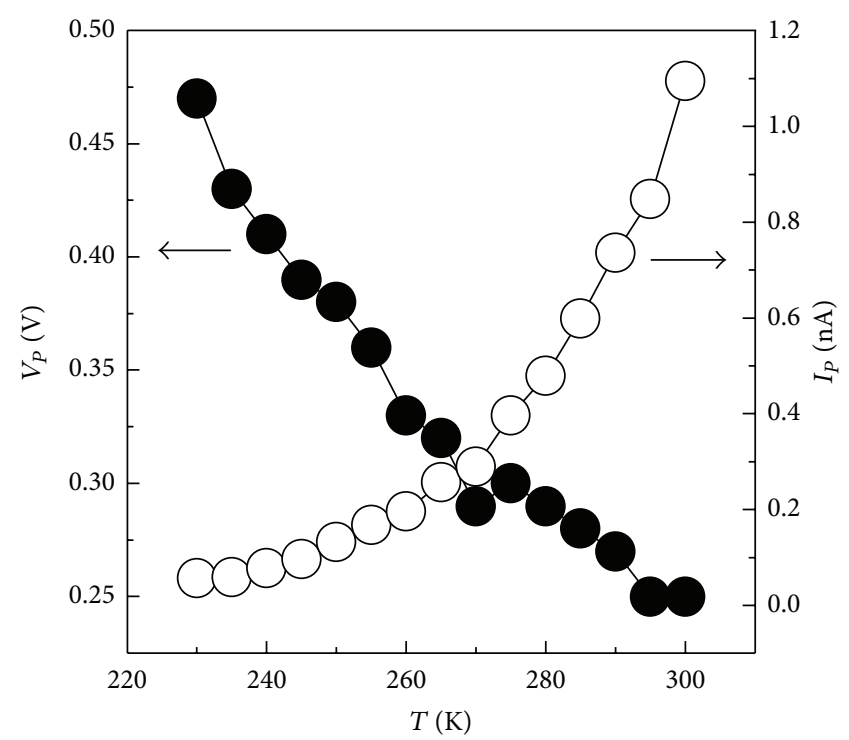

(b)

FIgURE 3: Abnormal NDR behavior. (a) Temperature-dependent resistivity follows the thermally activated hopping conduction (inset). (b) Contrary to NDR behavior reported previously, $V_{P}$ increases but $I_{P}$ decreases as the temperature decreases.

phenomena of the NSC crystal. Therefore, we suggest another possible mechanism for the NDR behavior.

Above all, we focused on the temperature-dependent NDR phenomena. Upon cooling, the values $I_{P}$ decreased but $V_{P}$ increased (Figure 3(b)). The temperature dependence of $I_{P}$ and $V_{P}$ was different from that of other NDR phenomena observed in the tunneling diode, Gunn effect [22], or subband transition from the high mobility of a fundamental level to the low mobility of a higher subband level, where $I_{P}$ increased and $V_{P}$ decreased upon cooling [9]. In case of a molecule containing a nitroamine redox center, both the $I_{P}$ and $V_{P}$ increased upon cooling [11]. The $I_{P}$ decrement of the NSC crystal upon cooling is attributed to the thermally activated hopping conduction as described in Figure 3(a). However, the $V_{P}$ increment on cooling is difficult to understand. We suggest a possible mechanism based on thermally modulated capacitance effect of the NSC itself.

At the beginning of voltage sweeping, the current increases due to the bias voltage and then charges are accumulated in the NSC gradually like a capacitor. At $V_{P}$, the charges are saturated in the NSC resulting in a drastic drop of the current at $V_{V}$. For $V>V_{V}$, the current exponentially increases with the increase of the bias voltage. This is called a thermal current [22]. During backward sweeping from $V_{V}$ to $0 \mathrm{~V}$, the current decreases exponentially, which follows the trace of a thermal current. In this process, hysteresis and discharging occur. As a negative bias voltage is applied, the same behavior is exhibited with an opposite polarity of charge. This results in symmetric NDR behavior with respect to the bias voltage.

In order to confirm that the charge storage is the dominant factor for the NDR of the NSC, we applied a different voltage $\left(V_{I}\right)$ at the beginning of sweeping (Figure 4(a)). When
$V_{I}$ started from the negative voltage (black line, from $-1.0 \mathrm{~V}$ to $+1.0 \mathrm{~V}$ ), the peak in the negative voltage region did not appear. On the other hand, when the $V_{I}$ was positive (red line, from $+1.0 \mathrm{~V}$ to $-1.0 \mathrm{~V}$ ), the peak in the positive voltage region was not exhibited. Nonezero $V_{I}$ over $V_{P}$ means that a large number of charges are already stored in the NSC. Therefore, NDR was not observed. This is similar to the previous literature on $\mathrm{ZnO}$ nanowires [23]. In case of a consecutive sweep from $-1.0 \mathrm{~V}$ to $+1.0 \mathrm{~V}$ and to $-1.0 \mathrm{~V}$, we found two peaks as expected (Figure 4(b)).

The decrease of $V_{P}$ with the temperature can be understood by the thermally activated mobility, $\mu=$ $\mu_{0} \exp \left(-\Delta / k_{B} T\right)$ [24]; here, $\mu_{0}$ and $\Delta$ are the prefactor and activation energy, respectively. This indicates that the mobility increases exponentially with the temperature and charges are more easily moved. At low temperature, an additional electrical potential, compared with high temperature, is necessary to fully store the charge in the sample. Hence, the $V_{P}$ increases as the temperature decreases.

\section{Conclusions}

In summary, the temperature-dependent symmetric NDR phenomenon in the NSC crystal has been investigated. As the temperature decreases, the $V_{P}$ increases but $I_{P}$ decreases. We consider the thermally modulated capacitance effect of the NSC as the origin of the NDR behavior.

\section{Acknowledgment}

This work was supported by the Incheon National University Research Grant in 20130418. 


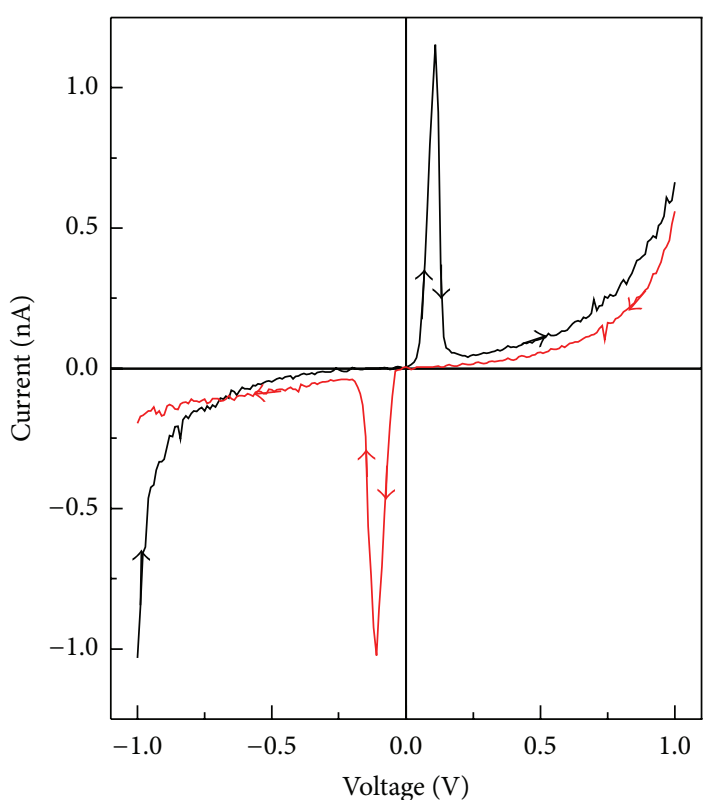

(a)

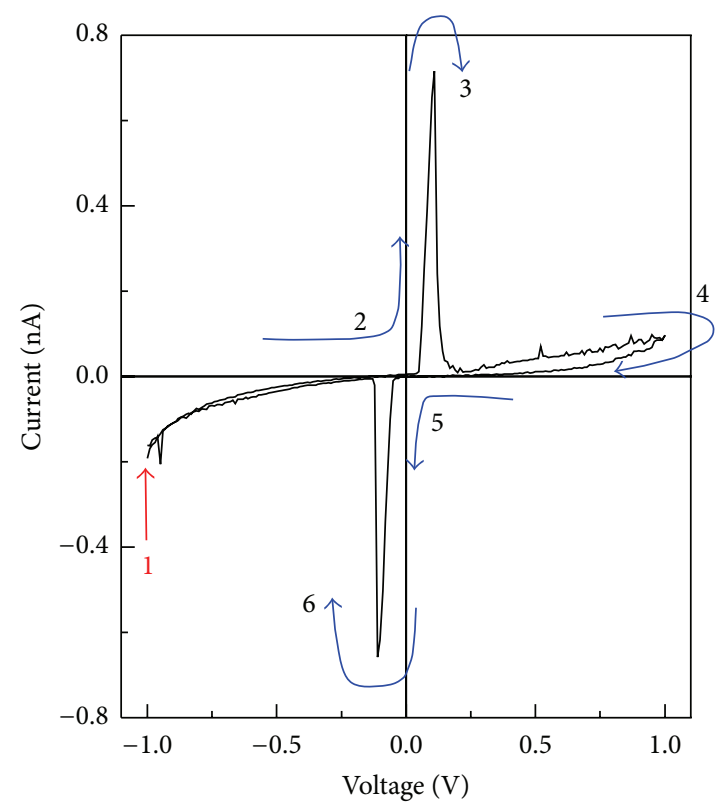

(b)

FIGURE 4: Capacitance effect of an NSC crystal. (a) A nonzero initial voltage results in the nonexistence of the peak in the voltage region, which is the same sign to the initial voltage. Black and red lines are the voltage sweep with negative and positive initial voltages, respectively. (b) $I-V$ characteristics were obtained by a consecutive bias voltage sweep. The number shows the sweep procedure.

\section{References}

[1] L. Esaki, "New phenomenon in narrow germanium p-n junctions," Physical Review, vol. 109, no. 2, pp. 603-604, 1958.

[2] L. L. Chang, L. Esaki, and R. Tsu, "Resonant tunneling in semiconductor double barriers," Applied Physics Letters, vol. 24, no. 12, pp. 593-595, 1974.

[3] M. A. Littlejohn, J. R. Hauser, and T. H. Glisson, "Monte Carlo calculation of the velocity-field relationship for gallium nitride," Applied Physics Letters, vol. 26, no. 11, pp. 625-627, 1975.

[4] J. R. Söderström, D. H. Chow, and T. C. McGill, "New negative differential resistance device based on resonant interband tunneling," Applied Physics Letters, vol. 55, no. 11, pp. 1094-1096, 1989.

[5] C. L. Wu, W. C. Hsu, M. S. Tsai, and H. M. Shieh, "Very strong negative differential resistance real-space transfer transistor using a multiple $\delta$-doping GaAs/InGaAs pseudomorphic heterostructure," Applied Physics Letters, vol. 66, no. 6, pp. 739741, 1995.

[6] T. Sugaya, K.-Y. Jang, C.-K. Hahn et al., "Enhanced peak-tovalley current ratio in InGaAs/InAlAs trench-type quantumwire negative differential resistance field-effect transistors," Journal of Applied Physics, vol. 97, no. 3, Article ID 034507, 2005.

[7] B. J. Keay, S. Zeuner, S. J. Allen Jr. et al., "Dynamic localization, absolute negative conductance, and stimulated, multiphoton emission in sequential resonant tunneling semiconductor superlattices," Physical Review Letters, vol. 75, no. 22, pp. 41024105, 1995.

[8] J. A. Switzer, B. M. Maune, E. R. Raub, and E. W. Bohannan, "Negative differential resistance in electrochemically selfassembled layered nanostructures," Journal of Physical Chemistry B, vol. 103, no. 3, pp. 397-398, 1999.
[9] B. Larade, J. Taylor, H. Mehrez, and H. Guo, "Conductance, I$\mathrm{V}$ curves, and negative differential resistance of carbon atomic wires," Physical Review B, vol. 64, no. 7, Article ID 075420, 10 pages, 2001.

[10] T. Wu and J. F. Mitchell, "Negative differential resistance in mesoscopic manganite structures," Applied Physics Letters, vol. 86, no. 25, Article ID 252505, pp. 1-3, 2005.

[11] J. Chen, M. A. Reed, A. M. Rawlett, and J. M. Tour, "Large on-off ratios and negative differential resistance in a molecular electronic device," Science, vol. 286, no. 5444, pp. 1550-1552, 1999.

[12] M. Galperin, M. A. Ratner, and A. Nitzan, "Hysteresis, switching, and negative differential resistance in molecular junctions: a polaron model," Nano Letters, vol. 5, no. 1, pp. 125-130, 2005.

[13] R. Liu, S.-H. Ke, H. U. Baranger, and W. Yang, "Negative differential resistance and hysteresis through an organometallic molecule from molecular-level crossing," Journal of the American Chemical Society, vol. 128, no. 19, pp. 6274-6275, 2006.

[14] H. Ren, Q.-X. Li, Y. Luo, and J. Yang, "Graphene nanoribbon as a negative differential resistance device," Applied Physics Letters, vol. 94, no. 17, Article ID 173110, 3 pages, 2009.

[15] X. F. Li, L. L. Wang, K. Q. Chen, and Y. Luo, "Strong current polarization and negative differential resistance in chiral graphene nanoribbons with reconstructed $(2,1)$-edges," Applied Physics Letters, vol. 101, no. 7, Article ID 073101, 5 pages, 2012.

[16] M. G. Silly, F. Charra, and P. Soukiassian, "Negative differential resistance at AgSi nanowires on silicon carbide: from a passive to an active massively parallel architecture," Applied Physics Letters, vol. 91, no. 22, Article ID 223111, 3 pages, 2007.

[17] H. R. Moon, C. H. Choi, and M. P. Suh, "A stair-shaped molecular silver(0) chain," Angewandte Chemie, vol. 47, no. 44, pp. 8390-8393, 2008. 
[18] K. Hariharan, "Electrical ionic conductivity and electronic spectra of superionic $\mathrm{KAg}_{4} \mathrm{I}_{5}$ films," Journal of Physics D, vol. 12, no. 11, pp. 1909-1916, 1979.

[19] A. Avogadro, S. Manzini, and M. Villa, "Ionic conductivity and disorder modes in borate glass systems," Physical Review Letters, vol. 44, no. 4, pp. 256-258, 1980.

[20] P. Vashishta, I. Ebbsjö, and K. Sköld, "Ionic motion in superionic $\mathrm{Ag}_{2}$ S," Journal of Physics C, vol. 18, no. 11, pp. L291-L296, 1985.

[21] M. A. Ureña, A. A. Piarristeguy, M. Fontana, and B. Arcondo, "Ionic conductivity $\left(\mathrm{Ag}^{+}\right)$in AgGeSe glasses," Solid State Ionics, vol. 176, no. 5-6, pp. 505-512, 2005.

[22] S. M. Sze, Physics of Semiconductor Devices, chapter 11, John Wiley \& Sons, New York, NY, USA, 2nd edition, 1981.

[23] Y. Zhang and C.-T. Lee, "Negative differential resistance in $\mathrm{ZnO}$ nanowires bridging two metallic electrodes," Nanoscale Research Letters, vol. 5, no. 9, pp. 1492-1495, 2010.

[24] P. W. M. Blom, M. J. M. de Jong, and M. G. van Munster, "Electric-field and temperature dependence of the hole mobility in poly(p-phenylene vinylene)," Physical Review B, vol. 55, no. 2, pp. R656-R659, 1997. 

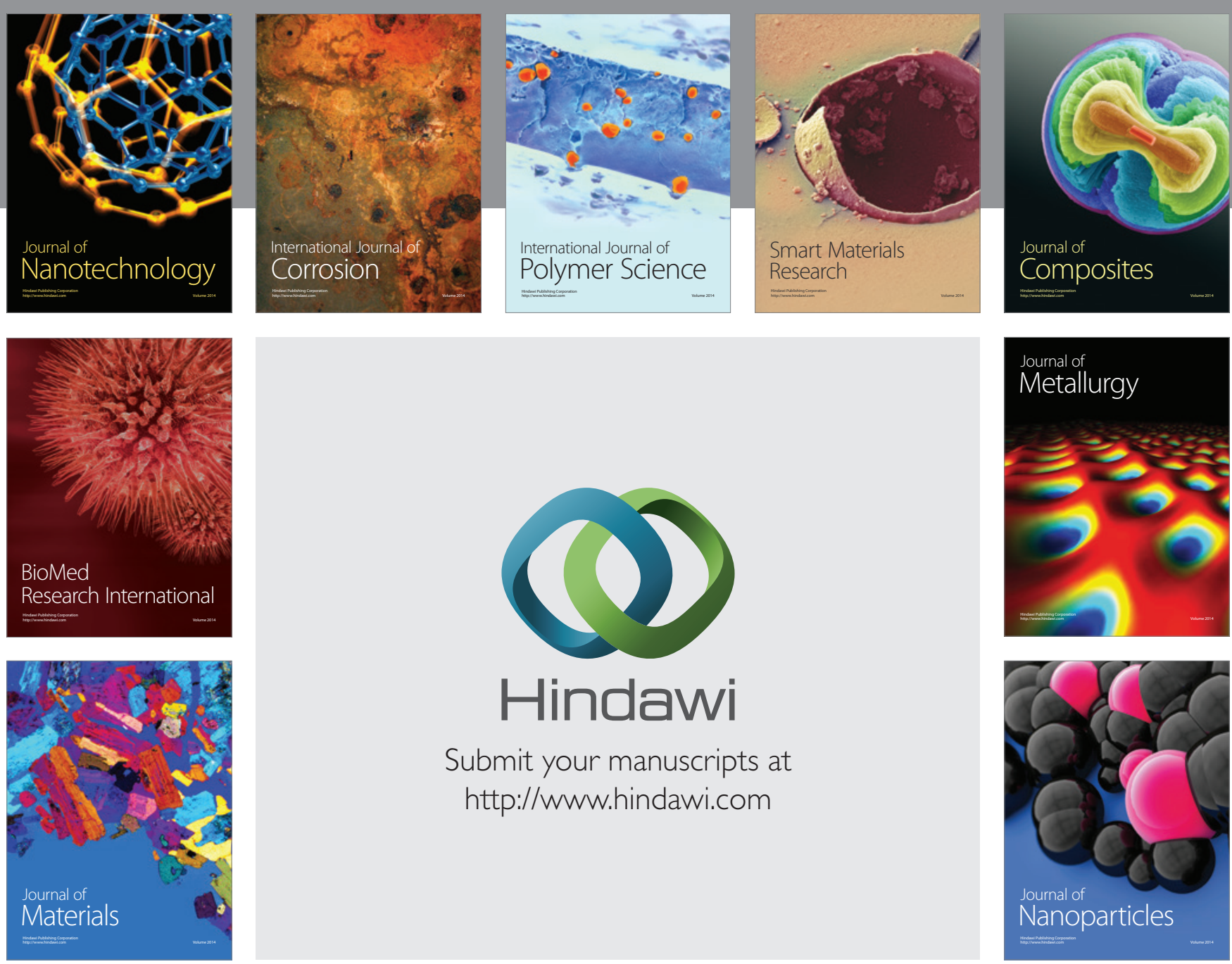

Submit your manuscripts at http://www.hindawi.com
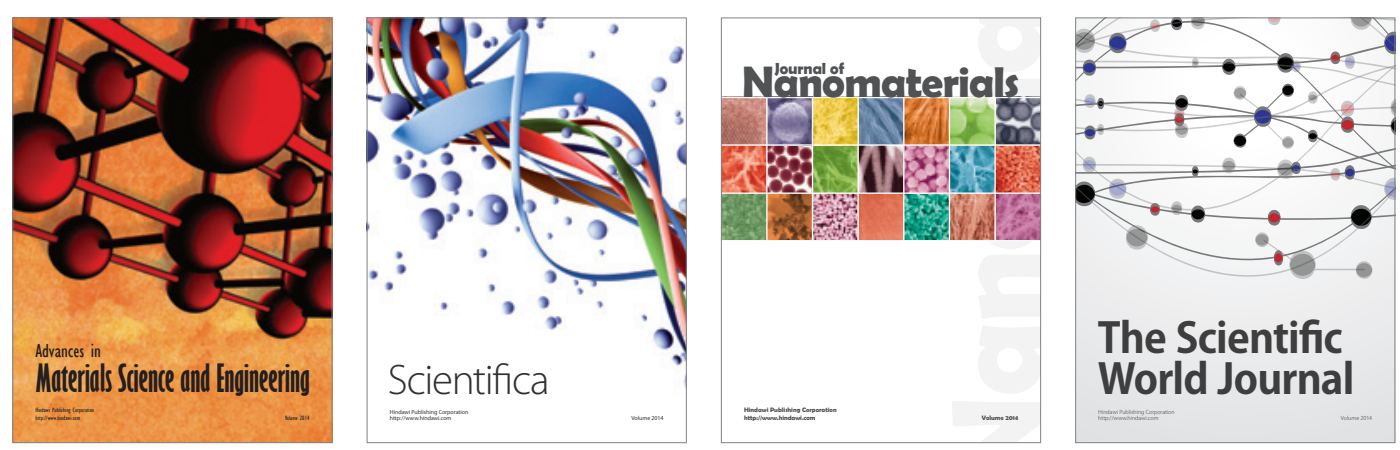

\section{The Scientific World Journal}
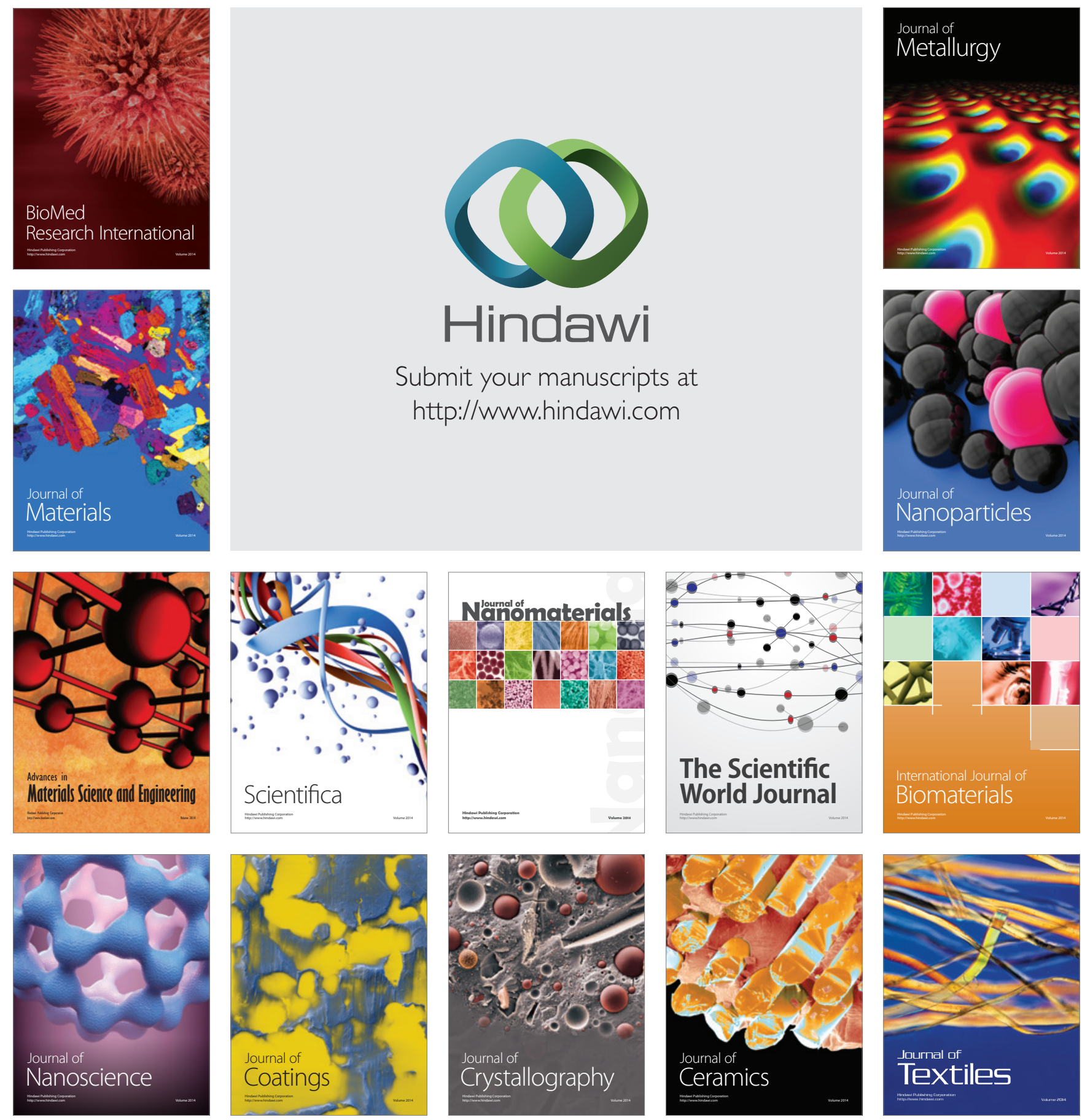